\title{
Beitrag zur Frage des Primäraffektes bei der Tuberkulose.
}

Von

\author{
Dr. Philipp S. Chancellor (Chicago). \\ (From the Otho Sprague Institute of the Childrens Memorial Hospital Chicaga.) \\ Mit 1 Tatel.
}

(Eingegangen am 18. Oktober 1913.)

Die Kenntnis der Einbruchspforte einer Infektion in den menschlichen Organismus hat nicht nur klinische Bedeutung. Sie vermag uns Aufschluß zu geben über die Art und Weise, wie der Infektionserreger in den Organismus eindringt und welche Wege dann dieser in ihm selbst nimmt. Glejchzeitig werden damit wichtige Anhaltspunkte in die Hand gegeben, wie wir eine Infektionserkrankung eventuell verhüten können.

Die Einbruchspforte bei der Syphilis, das Uleus durum, ist beinahe so lange bekannt, als diese Erkrankung existiert. Daß aber bei der Tuberkulose dieselben Gesetze obwalten, ist uns erst durch die Arbeiten Albreohts, Ghons, Hamburgers und Pirquets geläufig geworden. Der Grund für die Unkenntnis dieser wichtigen Tatsache liegt wohl darin, daß der Primüraffekt bei der Tuberkulose ganz verdeckt liegt und unserer klinischen Diagnostik nicht zugänglich ist. Nur in wenigen Fällen können wir den Primäraffekt bei der Tuberkulose intra vitam sehen und diagnostizieren. Da sind nur jene Falle, bei denen die Erstinfektion des Organismus auf der außeren Haut oder den der Inspektion zngänglichen Schleimhäuten statt hat. Die Literatur über diese Fülle ist. äußerst spärlich, besonders wenn man damit die so enorm angeschwollene Tuberkuloseliteratur vergleicht. Vor allem gehören hierher die Arbeiten, welche sich mit der Tuberkuloseinfektion bei der rituellen Besebneidung befassen. In jüngster Zeit berichtete Holt über einen derartigen Fall. In dieser Arbeit findet sich gleichzeitig die ganze Literatur darüber zusammengestellt, so daß ich hier nicht näher darauf einzugehen brauche. Weiter finde ich eine Mitteilung Ebsteins, welcher Tuberkuloseinfektion im Anschluß an eine Durehstechung des Ohrläppchens sah. 
Ich will zunächst über drei Falle berichten, von denen ich zwei längere Zeit beobachten konnte, während der dritte Fall mir durch eine mündliche Mitteilung J. B. Murphys, Chicago, zur Verfügung gestell wurde.

I. Mildred R. 5 Monate alt. Vater gesund, Mutter hat seit sieben Jahren Magenbeschwerden. Ein anderes Kind gesund.

Geburt normal. Kind bei Geburt normal entwickelt. Gewioht $41 / 4 \mathrm{~kg}$. Brust. ernuhturung mit zwei mohrwbchentlichen Untexbrechungen dureh 4 Monate. Das Kind war diese ganze Zeit über gesund, nahm zu.

Im Alter von 2 Monaten entwickelte sich auf der linken Wange eine braunxote Ffflorescenz, welche rasch größer wurde und dann exco. rijerte and geschwürsartig w urde. Dieses Geschwür wurde von verschiedenen Arzten mit allen möglichen Mitteln behandelt, ohne daß irgendein Erfolg zu ver. zeichnen gewesen wäre.

Das Kind war sonst bis zur Autnahme gesund, der Appetit immer gut, der Stuhl in Ordnung. 28. Februar Aufnahme in das Spital.

Status praesens: Gutexnahrtes kraftiges Kind. Vordere Fontanelle fur $2^{2} / 2$ Finger offen. Abdomen aufgetrieben, gespannt, Leber und Milz nicht palpabel. Auf der linken Wange ist eine ca. $1 \frac{1}{2}$ cm breite rote Papel zu sehen, deren Mitte goschwìrsartig verändert und mit einer dünnen weiben Kruste bedecktist. Unter dieser Efflorescenz ist eine zweite kloinere, hellrote, inflitrierte stelle zu sehen, oa. $2 \mathrm{~mm}$ im Durohmesser. Die Drüsen dor linken Halsseite stark geschwollen (Tafal).

Die Lungen zeigen normalen Befund. Eine Röntgenaufnahme der Lunge zeigt nichts Abnormales.

2. März Pirquet pos.

6. Märr. Wassermann neg.

10. Nürz zur Ergänzung der Anamnese.

Im November 1912 war die Muter krank, Das Kind wurde der Pflege einer Nurse uberatwortet, welche eimen ohronischen Hosten hatte and sehr viol expektorierte. Diese Nurse liebloste das Kind sehr of und biß es einmal in die Wage. Ebenso kostete sie die Nahrung des Kindes immer vor der Hahlzeit mit demselben Löffel, mit dem das Kind die Nahrung bekam. 5 Wochen nachher bemerkte die Hutter eine kileine gerötete Stelle an der linken Wange, wolche immer gröbex wurde. Das Sputum der Nurse wurde untersucht und in diescm konnten enorme Mengen von Tuberkelbacillen nachgewiesen werden.

17. März, Algemeinbefinden mveräudert. Das Abdomen stawk aufgetrieben und gespant. Milz sehr hart, a Querfinger untw dem Rippenbogen tastbar.

18. Màrz. Die Schwellung nnter dem linken Kinn zeigt die Große oiner WallnuB. Die Haut darüber gerötet. Die Schwellung wurde inzidicrt und eine groBo Menge Eiter entleert. In diesem Eiter konnten keine Tuberkelbacillen nach. gewicsen werben auch nittels der Antiforminnebhode nicht. THerversuch jedoch positiv.

22. Mär. Ubor dem linken Oberlappen ist bei der lerkussion eine letohte Dämpfung nachzwweisen. Auskultatorisch darüber nichts Abnownales zu finden. 
Die ganze Zeit iber blieb das Gewicht auf gleicher Höhe, ohne daß eine Znoder Abnahme erfolgte. Die Temperatur stieg böchstens bis $37,5^{\circ} \mathrm{C}$. Appetit and Strahl war immer in Crdnung.

14. Aprit. Das Kind wurde für einige Tage nach Hanse genommen und wird jetzt neverlich zur Spitalsbehandung gobracht. Das Kind war zu Hause ziemlich umuhig, 3-4 Stinle in Tag, welohe blutig gewesen sein sollen. Der Allgemeinbefund erscheint ziemlich der gleiche wie bei der Entlassung. Die Schwcllung am Halse reigt keino Veründerung, do Sokretion jedooh hat aufgehört. Dio beiden Infiltrationen im Gesichte ohne Veränderung. Die Lungen crgeben außer einer leichten Dämpfung übor dem linken Oberlappen nichts Auffallendes.

28. April. Die Läsionen im Gesichte heute alut entzindet. Die Mitte ist mehr dunkelcyanotisch, die Peripher aknt hellrot. Auch ist eine deutliche Verbreitung der. Entzünding zu konstatieren. Die Drüsen im Kieferwinkel sowie die Cervicaldrüsen stark geschwollen.

15. Mai. Das Kind wird nach Hanse genommen.

Der Allgemeinzustand hat sich beinahe nicht verändert. Gewicht ist dasselbe geblieben. Kein Temperaturanstieg.

II. Frances P, 7 Monate alt. Vater und Muttor leben.

Der Vatex leidet an einem chronischen Husten, sient sehr blaß aus und hat die beiden letzten Jahre sohr viel an Gowicht verloren. (Die Untersuchung seines Sputums ergah in jedem (xesichtsfolde sehr viele Tuberkelbacillen.) Die Nutter ist gesund. Zwei andere Söhne sind gesund. Die Geburt des Patienten war normal. Das Kind bei der Geburt gesund und kräftig. Das Kind bekam bis zur Aufnahme nur Brustmilch. Bis jetat keine Zahne. Bis zun 3. Monat entwickelte sioh das Kind gat und war nie krank. Darn bemerkten die Gltern, daß die rechte Halsseite anschwoll, and nach einem Monat wurde diese Geschwulst von einem Arzte geöffuet und es entleerte sich reichlich Eiter. Dann schloß sich die Fistel wieder and 6 Wochen daranf wurde eine newerliche Incision vorgenommen. Das Kind hatte von Zeit zu Zeit Temperatursteigerungen. Nie Husten. Der Appetit and Sehlaf war gut. Keine Krämpfe. Manchmal Frbrechen nach der Mahzeit.

Zur: Zeit der Aufnahme reigte das Lind folgenden Befund:

Abgemagert. Waut trocken. Parniculus adinosus sehr dinttig. Die vordere Fontanelle offen. Brust: Bei der Perhassion zeigt sich eine leichto Dämping üher dex reohten Seite. Die Atmung uber dieser war schux, besonders iber der roohten Spitize.

Herz: nomal.

Ablomen and Fxtremitituen: nomal.

Hats: The ganzen rechten torderen mal suthohen Halspartien stark geselwollen. Dio Schwelnng ist zürmlich hart und besteht aus einzeluen, gröberen Drüsen. Winige yon diesen fluktueren. An zwei stellen sind Narben ron rorangegangenen Incisionen sichtbar. An einer Stelle einese zernierente Fistel. Die ganze Schwellung erstreckt sich fon der Mittellinie rorn bis unter das rechte Ohr. Dio Halslympahdrüsen der linkrn Seite sind etwas geseluwollen.

Leber nioht palpabel, Milz 2 Querfingex unter dem Rippenbogen, sehr hart.

9. Tuli. Keine Veränderung. Erbricht nach dor Nahrungsaufuahme.

13. Juli. Eine genane Juspoktion des Rachens, welche nicht leicht möglich war, ergal, folgenden Befund. Der rechte weiche Gaumen ist vorgewälbt, 
gerötet. Bei Betastung ergibt sich, daB die Schwellung ziemlich hart ist und keine Fluktuation zeigt. Die Schleimhat der rechten Tonsille a ufgelockert und macht cinengeschwürsartigou Eindruck. Nase, sowcit zu examinieren, ist normal und zeigt keine Sekretion.

Pirquetreaktion positiv.

16. Juli. Der Allgemeinzustand hat sich plötzlich verschlimmert. Das Kind liegt in opistotonischer Stellung. Die Augen sind starr nach links geriohtet. BemuBtos. Die vordere Fontanelle vorgewölbt. Geringe Rigidität des Nackens. Dermographie pos. Kernig neg. Rachen wie oben, ehenso Drüsen.

Cheyne-Stockesche Atmung.

Präparate von den Tonsillen und der Nase ergehen negativen Befund bezüglich der Tuberkelhacillen.

Die Lumbalpunktion ergibt cine anschcinend klare Flüssigkeit unter einem atwas erhöhten Druck. Leukocythenzählung: 15 per $\mathrm{cmm}$.

Noguchi: pos. Glycyltryptophan: pos.

17. Juli. Status idem.

19. Juli. Der Algemeinzustand rerseblimmert sich sehr rasch. Ständig tonisch-klonische Krämpfe in den Extremitäten. Leichto Facialisparese der Jinken Seite.

Nahrungsaufnahme verweigert. Sondenfütterung. Konstipiert.

22. Juli. Exitus Ietalis.

Die Temperatur war wäbrend des 14 tägigen Spitaluufenthaltes ständig hoch, nur die letzten 21 Stunden war die Temperatur subnormal.

Im Trin wurde nichts dbnormales gefunden.

Sektionsbefund.

Ziemlich gnt genährtes und entwickeltes Kind.

Auf der rechten seite des Falses cin großer Tumon mit zahlreichen teils offenen, teils geschlossenen Fisteln. Bei Druck entleert sich aus den offenen Fisteln Eiter.

(Die Sektion konnte nur durch eine Offnung am Halse vorgenommen werden, da die Erlaubnis zur Öffnung des Bauches und der Brust nicht erhalten werden konnte.)

Durch Präparation drs Halses wurde ein großes Paket von Drüsen bloßgelegt, welches in ein dichtes, entzündich infiltriertes Gewebe eingebettet ist. Den größten Teil dieses Drüsenpaletes bildon zwei rolstündig vorkäste Drüsen von opaln gelber Farbe. Von ritser stelle weiter nach abwärts rieht, sioh eine Kette von immer kleiner werdenden Drüsen hin, welche auf $\mathrm{km}$ Durchschnitt ebenfalls kloincre und gröere rerkäste Knütchen zeigen. Dio Drüsen an der linken Halsseite zeigen makroskopisch keine tuberkulösen Veränderungen. Die Jungen, das Herz und die übrigen mediastinalen Gobilde wurden durch die Incision am Halse entfernt. Dir modiatiualen un bronchialen Lxmphutusen zeigen eino geringe Vergröberung, keine Verkäsmg. In den Inngen sind gröbere und kleinere miliare Tuberkel zu finden. Es ist whoh trotz genaner Durchsuchung aller Lungenpartien kein Primärherd nachzuweisen. Dic rechte Tonsille vergrößert, zeigt eine größère Uleeration. Die linke Tonsillo normal.

Horz negativ. Die Buacheingeweide konnten nicht entfernt werden.

Th. Nach einer persöntichen Mitteilmg .. B. Auxpbys, Chicago, Iann ich uber folgenden Fall berichten: 
Ein ca. 1 Jahr alter Knabe zeigte a uf einer Wange eine etwa erhsengroße Infiltration mit rogionärer Schwellung der Drüsen am Halse. Die Infiltration brach später auf und wurde geschwürartig. Die Therapie war erfolglos. Die Anamnese dieses Falles ergibt, daß zwar die Eltern dieses Kindes gesund waren, daß aber ein Onkel an einer progressiven Phtise litt. Diesor Onkel hatte die Gowohnheit, smmex eine Stecknardel im Munde zi haben. Er war sehr häufig mit dem Kinde zusammen und war sehr zärtlich mit ihn. Eines Tages küBte er das Kind und verletzte mit der ständig im Mnude befindlichen Stecknadel die Wange des Kintes, so daß es blutete. (a. 1 Wonat darauf wurde die gerotete Hantstelle bemexkt, dì dann später zu dem oben beschriebenen (xeschwür wurde.

Die Untersuchmg des Sputums dieses Mannes ergab die Anwesenheit von sohr zahlreichen Tuberkelbacilen. Also anch in diesem Falle waven Tuherkolbacillen durch die verletzte Haut duchgedrungen, hatten sich angestedels and den Primäraffels gesotzt.

Ich fasse num die aus diesen Krankengeschichten sich ergebenden Tatsachen zusammen. Vor allem will ich hier erörtern, wie die Diagnose gestellt wurde. Bei den ersten zwei Fallen ergab die positive Cutanreaktion, dab die Kinder bereits tuberkalos infiziert waren. Da eine positive Cutanreaktion bei Kindern unter: 1 Jahr stcts auf einen aktiveu ProzeB hinweist, so war damit schon cin wichtiger Anhaltspunkt für die Dia gnose gegeben. Des weiteren bot der Primäraffekt als solcher ein ganz charakteristisches Bild. Tin Fall I sahen wir eino sich allmählich vorgrößernde Infiltration auf der Wange, die später exulcerierte. Diese ständig fortschreitende Entzündung, dje jeder Behandlung trotzte, wies darauf hin, daß es sich nicht um oinen pathologischen ProzeB handeln kounte, wic wir ihn bei der Infektion mit den gewöhnlichen Bakterien. wis Staphylokokken oder Streptokokken, sich entwickeln sehen, sondern, aaß es sich um einen chronischen ProzoB handeln muBte. Ganz ahnlich waren die Erwagugen in Fall $\mathrm{ll}$. Im Fall II bot das mur schwer zu sehende Goschwur aul der Tonsille schon mehr Sohwierigkeiten. aber ancl hior lag es nahe, da wir chronische Gegohwire im Xasenrachenraum auBer Tuberkulose und Syphilis nicht kennen, die Diagnose auf Tuberkulose zu stellen. Einen weiteron Anhaltspunkt für die Diagnose gahen wn die regionaren Drisenschwellungen. Die Tuherkalose dringt ganz gesetanäbig ant dem Wege der Lymphbahn im Körper vor. so zwar, daß dio nächstgelegenen Iymphdrüsen an ersten befallon werlen wn daf diese auch weiterhin die staxksten Varanderungen anfweisen, wie besonders durch die Arbeten Ghons und Hamburgers nachgewiesen wưde. Wir sahen, daß die Drüsen vereiterten, und im Tall I zeigte der Mcerschweinohenversuch, dab dcr Eiter Tuberkchbacillen enthielt. Einen weiteren Hinweis fiu die Diagnose ergab die 
Anamnese. Bei allen 3 Fällen konnte nachgewiesen werden, daß schwere Phthisiker, die massenhaft Tuberkelbacillen in ihrem Sputum beherbergten, ständig mit den Kindern in Berührung waren. Bezüglich des klinischen Verlaufes dieser Fälle ist zusammenfassend folgendes zu sagen: Die Kinder in den Fällen I und II zeigten lange Zeit einen ziemlich guten Ernährungszustand. Fall I hatte zwar die ganze Beobachtungszeit über keine Gewichtszunahme zu verzejchnen, aber auch eine Abnahme war nicht zu kcnstatieren. Im Fall II war der Ernährungszustand auch ein ziemlich guter, jedoch verursachte die aufretende miliare Tubcrkulose einen plotzlichen und rapid zunehmenden Vertall und dann den Exitus des Kindes. Die Lange der. Erkrankungsdauer ist auch in den beiden exsten Fallen bemerkenswert. Fall I lebt noch jetzt, also nach einer 6 monatichen Krankbeitsdauer, während Fall II ca. 4 Monate die Erstinfektion überlebte. Besonders der Fall I zeigte daher eine ungewöhnlich lange Krankheitsdaucr, ja es ist sogar nicht ausgeschlossen, daß das Kind vollkommen diese Infektion übersteht. Auch der Fall II zeigte lange Zeit hindurch keine besondere Alteration des Allgemeinbefindens, und auch hier hat nur die plötzlich auftretende miliare Tuberkulose den Exitus herbeigeführt. Vergleicht man diese beiden Fäle wit den Fällen von Säuglingstuberkulose, dessen Primär herd in den Lungex liegt, so zeigen sich Keine hesonderen Untersohiede. Auch in den letztgenannten wallen finden wir, soweit überhaupt festzustellen ist, längere und kürere Erkrankungsdauer, ebenso mehr oder minder guten Ermähungszustand und als Schlub den Exitus, horvorgorufen duroh eine miliare Tuberkulose oder auch therleben des 2. und 3. Lebensjahres, ja auch vollstinndiges Uberleben der Infektion. Was den Infektionsmodus anbelangt, so erhalten wir durch diese Fälle einen äuBerst interessanten AufschluB. Die Infektion eufolgte in dem. Fall I und III nicht durch die normale Hautdecke, sondern duroh eino ziemlich lädierte. Es geht also hervor, daß die Infektion mit Tuberkulose in dic abero Hantdecke nur dann erfolgt, wenn dieselbe nicht intakt ist. Dafür sprechon anch die in der Literatur gosammelten Fülle von Primäraffekt auf der äuberen Hautdecke. Ws dürtte anch ziemlich waturcheinlich sein, daß die Tuberkulosenfekton auch in den Schleimhäuten des Nasen- und Rachenrames nur dann haftet, wenn sich Läsionen in derselben befinden. Denn in andcren Falle wübten wir mehr Primaraffekte in Nasenrachenaume zu sehen bekommen.

Gleichzeitig sei auch darauf hingewiesen, das sich die oben bew schriebenen tuberkulösen Primäraffekte absolut von denen unterscheiden, 
bei denen zwar ebenso eine Tuberkuloseinfektion in die äußere Haut stattgefunden hat, wie bei Schlächtern, Anatomiedienern usw., die aber klinisch ganz anders aussieht, da es sich in diesen Fällen nicht um eine primäre Infektion mit Tuberkulose, sondern um eine Superinfektion mit Tuberkelbacillen handelt.

Auch diese Fälle zeigen wieder die Notwendigkeit, prophylaktisch eine Tuberkuloseinfektion zu verhüten. Es sind, wie immer wieder betont wird, aber leider immer noch nicht genug berücksichtigt wird, Phthisiker auf jeden Fall von Kindern, und ganz besonders von Kindern unter $1 \mathrm{Jahr}$, fernzuhalten.

Zusammenfassung: In der vorliegenden Arbeit werden 3 Fälle von tuberkulösen Primäraffekten bei Kindern unter einem Jahre beschrieben. Zwei von diesen befanden sich auf der äußeren Haut, und zwar auf der Wange, einer auf der Tonsille. Es wird auf die Literatur über dieses Thema hingewiesen, weiter die genauen Krankengeschichten wiedergegeben und die Diagnose besprochen.

\section{Literaturverzeichnis.}

1. Albrecht, H., Uber Tuberkulose des Kindesalters. Wiener klin. Woohensobr. 1909, Nr. 10.

2. Ghon, Der primäre Lungenherd bei der Tuberkulose der Kinder. Urban und Schwarzenberg 1912.

3. Hamburger, Allgemeine Pathologie und Diagnostik der Kindertuberkulose. Wien 1910. Deutike.

4. v. Pirquet, Lehrbuch der Kinderheilkunde von Feer. Artikel "Tuberkulose",

5. Holt, L. E., Tuberculosis acquired through ritual circumcision. Joum. of the Am. Med. Ass., Juli 12, 1913.

6. Epstein, A., Das Ohrringstechen und seine Gefahren usw. Zeitschr. R. Kinder. heilk. Kassowitz-Festachrift.

Chicago, Ill., U. S. A., 2732 Pine Grove Avenue. 


\section{after page 508}

Zeitsehrift für Kinderheilkunde. Orig. $\mathrm{X}$.

Tafol 1 .
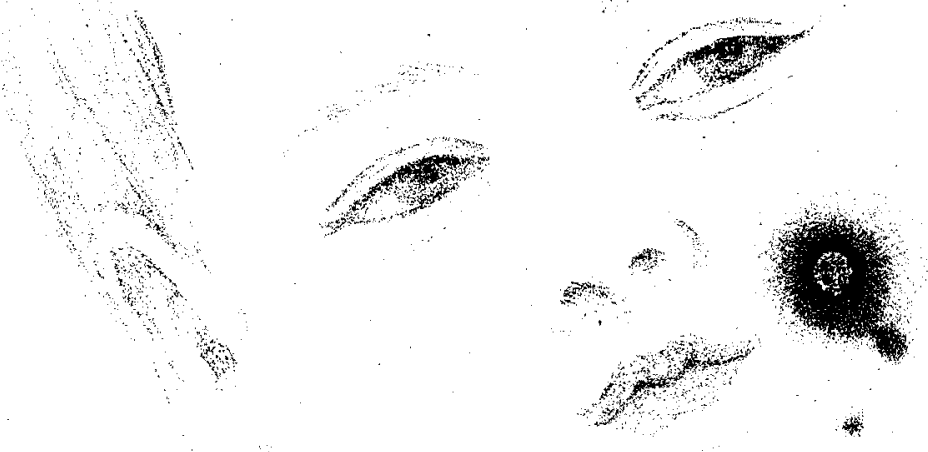

Zoichnung von Mrs. H. F. Heimholz, Evanstone. 